\title{
Evento de Intoxicación en Perros de Zona Urbana mediante Cebos Contaminados con Aldicarb
}

\author{
Poisoning Event in Dogs in an Urban Area Using Baits Impregnated \\ WITH AldicARB
}

\author{
Eliana L.R. Saldeña ${ }^{1}$, Valentina Hynes ${ }^{1}$, Daniela M. Ferré ${ }^{1,2}$, Martín Quero ${ }^{1,2}$, \\ Verónica Neuilly ${ }^{1,3}$, Nora B.M. Gorla ${ }^{1,2,3}$
}

\section{Resumen}

El objetivo de esta investigación fue implementar métodos de baja complejidad como la medición de actividad butirilcolinesterasa y cromatografía en capa fina $(\mathrm{CCF})$ para identificar el agente causal de un evento de intoxicación que afectó a 45 perros de una zona urbana de la ciudad de Mendoza, Argentina. Se identificó al plaguicida aldicarb en un cebo que fue extraído de la boca de uno de los perros intoxicados. Aldicarb es un plaguicida carbamato anticolinesterásico de alta toxicidad, baja seguridad al manejo, con efectos ecotoxicológicos y con limitaciones edáficas y de uso en Argentina desde 1990. Los niveles de actividad colinesterasa fueron bajos e indicadores informativos de la evolución o desenlace de los animales.

Palabras clave: perros intoxicados; aldicarb; colinesterasa; cromatografía en capa fina; CCF; carbamato; cebos envenenados

\section{Abstract}

The aim of this study was to implement low complexity methods as butyril cholinesterase activity and thin layer chromatography (TLC) to identify the causal agent of an event that affected 45 poisoned dogs in Mendoza, Argentina. The pesticide identified in the bait extracted from the mouth of a canine was the insecticide carbamate aldicarb,

\footnotetext{
${ }^{1}$ Facultad de Ciencias Veterinarias y Ambientales, Universidad Juan Agustín Maza, Mendoza, Argentina

${ }^{2}$ Consejo Nacional de Investigaciones Científicas y Técnicas - CONICET, Mendoza, Argentina

${ }^{3}$ Cuerpo Médico Forense y Criminalístico, Mendoza, Argentina

${ }^{4}$ E-mail: noragorla@gmail.com
}

Recibido: 7 de diciembre de 2016

Aceptado para publicación: 19 de mayo de 2017 
substance with high toxicity, low management safety, with ecotoxicological effects, and with restrictions for its use in Argentina since 1990. Cholinesterase activity levels were indicators of the outcome of the animals.

Key words: poisoned dogs; aldicarb; cholinesterase; thin layer chromatography; TLC; carbamate; poisoned baits

\section{INTRODUCCIÓN}

Las intoxicaciones intencionales en animales de compañía son una creciente problemática, no solo en Argentina sino en muchos otros países, donde los compuestos organofosforados (OF) y carbamatos (CB) son los más comúnmente utilizados (Berny et al., 2010; Caloni et al., 2016). En un estudio realizado en Italia, se reporta que los insecticidas y los rodenticidas causaron el 40.8 y $27.6 \%$ de los eventos de envenenamiento, respectivamente, afectando mayormente a perros $(71.1 \%)$ y gatos $(15.8 \%)$ y un número limitado de casos en caballos, cabras y ovejas (Caloni et al., 2016).

Reportes sobre intoxicaciones en animales de compañía demuestran que los compuestos principalmente utilizados con este fin son los CB en general y aldicarb en particular (Verster et al., 2004; Xavier et al., 2007; Berny et al., 2010; Caloni et al., 2016). A nivel local, Ferré et al. (2015) confirmaron que el insecticida fue el agente causal de dos eventos de intoxicación en perros en la ciudad de Mendoza, Argentina. En Argentina, no existen registros epidemiológicos de casos de animales intoxicados, como si ocurre con el Centro de Información y Asesoramiento Toxicológico (CIAT, Mendoza) para personas.

Debido al mecanismo de acción anticolinesterásico que presentan los $\mathrm{CB}$ y $\mathrm{OF}$, la medición de la actividad colinesterasa resulta útil en el diagnóstico, tratamiento y pronóstico del individuo intoxicado. En el diagnóstico, porque la actividad de la butirilco- linesterasa/ colinesterasa plasmática (BChE) refleja la actividad de la acetilcolinesterasa del Sistema Nervioso Central (SNC), responsable de la presentación de los signos (Groner et al., 2007); en el tratamiento, ya que si bien tanto OF como CB son agentes anticolinesterásicos, los signos del SNC son menos frecuentes en intoxicaciones con CB (Soler Rodríguez et al., 2006); y en el pronóstico, dado que la determinación de la actividad de $\mathrm{BChE}$ en el tiempo permite distinguir entre intoxicaciones causadas por compuestos $\mathrm{OF}$ y CB. Esta distinción resulta útil en la clínica, ya que es posible la expresión de un síndrome intermedio o neuropatía retardada en intoxicaciones por OF (Hopper et al., 2002; Cabrera et al., 2009). El individuo intoxicado presenta signos clínicos por estímulo muscarínico y nicotínico; sin embargo, la acumulación del neurotransmisor acetilcolina en las terminaciones sinápticas, principalmente muscarínicas, inicialmente excita y posteriormente inhibe la trasmisión en el SNC (Campbell y Chapman, 2000; Verster et al., 2004).

En la práctica veterinaria, el agente causal no es usualmente identificado dada la carencia de equipos de cromatografía líquida o gaseosa en los laboratorios veterinarios, lo cual dificulta las denuncias y registros oficiales de envenenamiento. El uso de técnicas de laboratorio para la medición de BChE y la cromatografía en capa fina son métodos factibles y de fácil acceso para su uso en medicina veterinaria. La implementación de estos métodos diagnósticos complementarios por parte de los profesionales podría ser la primera acción que aportaría a un futuro registro de intoxicaciones en la región. Esto resul- 
ta interesante en salud pública, pues facilita la identificación de agentes tóxicos que se encuentran a disponibilidad pública y que no son usados para los fines para los cuales fueron producidos.

El objetivo de este trabajo fue investigar un evento de 45 perros con signos de intoxicación compatibles con agentes anticolinesterásicos, que se registró en un lapso de una semana en una zona urbana de la ciudad de Mendoza.

\section{Materiales y Métodos}

\section{Ubicación del Estudio}

El evento de la intoxicación se presentó en el distrito El Bermejo (departamento de Guaymallén, Mendoza, Argentina) en diciembre de 2015. La zona ocupa una superficie de $5.25 \mathrm{~km}^{2}$ y cuenta con una población de 13428 habitantes, lo que resulta en una densidad de población de $2.6 \mathrm{hab} / \mathrm{km}^{2}$, densidad media comparada con los demás distritos del departamento (Sistema Estadístico Municipal, 2001).

El Bermejo forma parte del área complementaria denominada zona de Preservación Patrimonial. Se dispone de ordenanzas que regulan su crecimiento, protegiéndolo de actividades que actúen en detrimento de su medio ambiente. Así, la Ordenanza 224/1988 para «Preservar el entorno socio-económico y ecológico de la zona» y la Ordenanza 4893/ 1999 «Prohibición de la instalación y radicación, de usos o emprendimientos que degraden las características generales del sitio y que provoquen un impacto de magnitud sobre el medio ambiente y sobre las actividades y vida propia de determinadas áreas» (Ordenanzas del Honorable Consejo Deliberante, Guaymallén).

\section{Animales y Muestras}

- Grupo intoxicado: Formado por 36 caninos adultos con similares signos de intoxicación compatible con sobreestimulación nicotínica (sialorrea, lagrimeo, incontinencia urinaria, diarrea, bradicardia, broncoconstricción con aumento de secreciones bronquiales y miosis) y signos muscarínicos (temblores, rigidez muscular, debilidad y parálisis). Diez de estos animales (seis machos y cuatro hembras), tenían dueño y fueron asistidos clínicamente en dos veterinarias de la zona. A ocho de estos se les tomó una muestra de sangre para medir la actividad BChE, llegándose a recuperar exitosamente del cuadro agudo de intoxicación. Los 28 caninos restantes fueron hallados muertos en la vía pública y no se obtuvo información ni reclamos de su tenencia por algún propietario aparente.

- Grupo control: Formado por 13 caninos adultos sanos (nueve hembras y cuatro machos) que no habitaban en la zona de El Bermejo. Se les hizo una evaluación clínica para asegurar que presentaban un estado sanitario óptimo. Se les tomó muestras de sangre para la obtención de valores de referencia de la actividad BChE en caninos adultos de la región.

El tratamiento proporcionado a los animales consistió en fluidoterapia endovenosa con solución de cloruro de sodio al $0.9 \%$ y atropina $0.5 \mathrm{mg} / \mathrm{kg}$. El $50 \%$ de la dosis total se administró vía endovenosa y la mitad restante vía subcutánea. Cuando fue necesario controlar las mioclonías y lograr la relajación del animal, se administró $0.5 \mathrm{mg} / \mathrm{kg}$ de diazepan endovenoso a una concentración de $10 \mathrm{mg} / \mathrm{ml}$.

Las muestras de sangre $(1 \mathrm{ml})$ se colectaron por punción de la vena cefálica en tubos con $40 \mu 1$ de heparina sódica al 5\% (500 $\mathrm{UI} / \mathrm{ml})$. La sangre fue centrifugada el día de la colecta a $500 \mathrm{~g}$ durante $10 \mathrm{~min}$ y el plasma resultante fue almacenado a $-4{ }^{\circ} \mathrm{C}$. 
Cuadro 1. Valores de actividad butirilcolinesterasa plasmática (UI/l) (promedio \pm desviación estándar) en caninos con signos de intoxicación compatibles con agentes anticolinesterásicos (El Bermejo, Mendoza, Argentina)

\begin{tabular}{lcc}
\hline Grupo & \multicolumn{1}{c}{ Butirilcolinesterasa (UI/l) } \\
\hline Control $(\mathrm{n}=13)$ & $4390.7 \pm 1548.4^{\mathrm{a}}$ \\
Intoxicado & & $2607.9 \pm 1380.2^{\mathrm{b}}$ \\
& Sobrevivientes $(\mathrm{n}=8)$ & \\
$\quad$ Fallecidos $(\mathrm{n}=2)$ & $336.5 \pm 154.8$ \\
\hline
\end{tabular}

a,b Superíndices diferentes entre grupos indican diferencia estadística $(p=0.0092)$

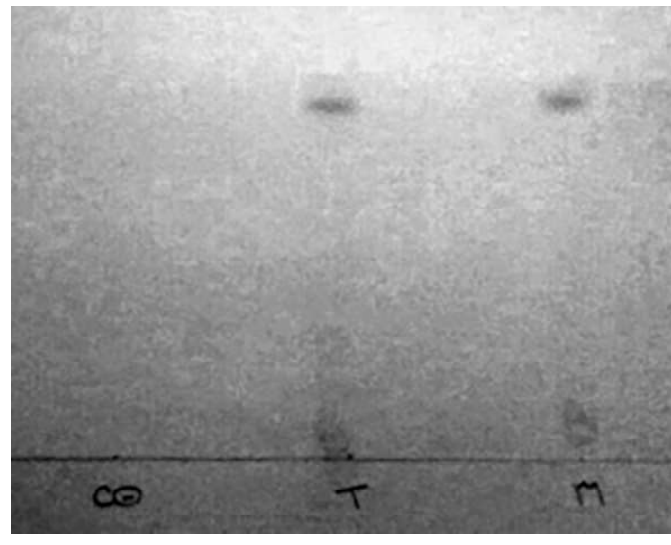

Figura 1. Placa de silicagel con revelador para carbamatos. C(-) control negativo, T: testigo de aldicarb, M: muestra, extracto de cebo contaminado

El cebo empleado para el envenenamiento de los canes fue extraído de la boca de uno de los animales del grupo de intoxicados y se mantuvo refrigerado a $-8^{\circ} \mathrm{C}$ en envase plástico con sello hermético hasta el momento del análisis. El cebo tenía apariencia de tejido animal, de tamaño $4 \times 4 \mathrm{~cm}$.

\section{Determinación de Butirilcolinesterasa}

Se determinaron los niveles de $\mathrm{BChE}$ plasmática utilizando el kit Colinesterasa (GT Lab $\left.{ }^{\circledR}\right)$, método cinético por espectrofotometría a $405 \mathrm{~nm}, \mathrm{pH} 7.7$ y $25^{\circ} \mathrm{C}$ (Ellman et al., 1961). La reacción utiliza butirilcolina como sustrato y se realizó a temperatura ambiente. En el kit, diseñado para medicina humana, se efectuó la reducción proporcional de los volúmenes, para hacer dos determinaciones por cada $3 \mathrm{ml}$ de sustrato butiriltiocolina provisto en cada vial. Los resultados fueron expresados en UI/1.

\section{Cromatografía en Capa Fina}

Se efectuó marcha toxicológica para tóxicos orgánicos fijos, con recuperación del extracto ácido y utilización de columnas EXtrelut ${ }^{\circledR} N T 3$ de $15 \mathrm{ml}$ según el manual de procedimientos analíticos toxicológicos para laboratorios de baja complejidad, con leves modificaciones (Flanagan et al., 1995). Para la Cromatografía en Capa Fina (CCF) se utilizaron las siguientes placas como adsorbentes: TLC Silicagel 60 F254 20x20 cm (Merck $\left.{ }^{\circledR}\right)$ y cuba vertical para cromatografía de $15 \times 15 \times 6$ cm impregnada con solvente de corrida cuádruple: n-hexano/ciclohexano/cloroformo/ acetona (40/40/10/10), a $25^{\circ} \mathrm{C}$. Se sembraron testigos estándares de plaguicidas $\mathrm{OF}$ (clorpirifos, metamidofos, metidatión), organoclorados y CB (aldicarb, carbaril, carbofuran y carbosulfan), y el extracto de la muestra en estudio. Se compararon las distancias recorridas por la muestra testigo y los estándares identificando la sustancia en la muestra en estudio cuando la distancia coincidía con la del testigo estándar conocido. 


\section{Análisis Estadístico}

Para el estudio de los datos de actividad BChE se utilizó el programa estadístico Prism 6.0. Se aplicó el test de KolmogorovSmirnov para comprobar el ajuste a una distribución normal y el t-Student para analizar la diferencia de actividad enzimática entre los grupos control e intoxicado $(\mathrm{p}<0.05)$.

\section{Resultados}

Los valores de actividad BChE de los animales del grupo control e intoxicado fueron estadísticamente diferentes $(p<0.01)$. Los ocho canes que recibieron atención médica presentaron un $30 \%$ de inhibición de la $\mathrm{BChE}$ $\mathrm{y}$ respondieron de forma favorable al tratamiento recuperándose del cuadro de intoxicación. Los dos animales del grupo intoxicado que tenían valores notoriamente disminuidos de actividad BChE ( $92 \%$ de inhibición) murieron.

En la placa de silicagel donde se sembró el extracto orgánico obtenido de la muestra de cebo contaminado se observó, mediante el vaporizado con el revelador de CB (4-nitro benceno diazonio-tetra-fluoroborato endietilén glicol/alcohol absoluto [1/9]), la presencia de una mancha que indica la presencia de aldicarb en la muestra estudiada. En la Figura 1 se observa que la distancia de recorrido de la muestra coincide con la del testigo aldicarb.

\section{Discusión}

Se identificó al insecticida carbamato aldicarb como el plaguicida presente en el cebo analizado. Este CB se utiliza en producción frutícola como acaricida, insecticida y nematicida, de acción por contacto y sistémica. Es extremadamente tóxico para peces, aves y mamíferos (Nelson et al., 2001). Los principales metabolitos biológicamente activos del aldicarb son el aldicarb sulfóxido, con 76 veces más actividad anticolinesterásica, y el aldicarb sulfona, el cual es 25 veces menos tóxico, pero con mayor persistencia en el organismo (Cazenave et al., 2005).

La Organización Mundial de la Salud lo clasifica toxicológicamente como extremadamente peligroso por lo que integra la lista de Pesticidas Altamente Peligrosos (HHP por sus siglas en inglés), para los que se recomienda un manejo particular (WHO, 2016). En la Unión Europea está prohibido el uso y la comercialización de aldicarb debido a su alta toxicidad, baja seguridad al manejo y efectos ecotoxicológicos (Ruiz-Suárez et al., 2015).

En Argentina, aldicarb tiene limitaciones edáficas y de uso (Decreto Nacional N. ${ }^{\circ}$ 2,1990 ), que limita su aplicación a personal profesional o capacitado; sin embargo, Hynes (2014), mediante encuestas a trabajadores de fincas frutícolas en la ciudad de Mendoza encontró que solo el $26 \%$ de los usuarios cuenta con asesoramiento profesional, que el $29 \%$ los adquiere directamente, y que muchos de ellos solo siguen las recomendaciones del Instituto Nacional de Tecnología Agropecuaria (INTA), del Instituto de Sanidad y Calidad Agropecuaria de Mendoza (ISCAMEN) o del Servicio Nacional de Sanidad y Calidad Agroalimentaria (SENASA).

Se han presentado casos accidentales de intoxicaciones en seres humanos al utilizar aldicarb como rodenticida (Lima y Reis, 1995). La DL 50 de aldicarb en caninos es de $6.5 \mathrm{mg} / \mathrm{kg}$, ocurriendo la muerte por falla respiratoria (Arnot et al., 2011). La DL en humanos es de $0.8 \mathrm{mg} / \mathrm{kg}$ (Waseem et al., 2010), de allí la importancia del control del uso indebido de estos tóxicos, al presentar el hombre mayor sensibilidad que otras especies. Aldicarb, además de ser usado como plaguicida, se sigue utilizando para intoxicaciones intencionales (Martínez-Haro et al., 2008; Anastasio y Sharp, 2011). 
En esta investigación se obtuvieron valores propios de referencia para la actividad BChE $(4390 \pm 1548 \mathrm{UI} / \mathrm{L}$ en caninos adultos machos y hembras) que, considerando los desvíos estándares, son comparables a las reportadas por otros autores, siguiendo la misma metodología: $3276 \pm 845$ y $3013 \pm 1081$ para machos y hembras, respectivamente (Maia et al., 2012) y $5071 \pm 891$ UI/L (Evans, 1990).

La actividad $\mathrm{BChE}$ tiene un amplio rango de variabilidad en animales clínicamente sanos, pudiendo alcanzar niveles del $30 \%$. Debido a esto es la conveniencia de obtener valores de referencia propios en cada laboratorio (Maia et al., 2012). Los valores de referencia propios pueden aplicarse en el diagnóstico diferencial entre OF y CB. Así, una segunda determinación a las $24 \mathrm{~h}$ puede inferir la presencia de un OF si se mantiene el nivel de inhibición o de un CB si la actividad enzimática ha aumentado, ya que los primeros producen una inhibición irreversible de la BChe y los segundos una inhibición reversible (Colovic et al., 2013).

Tanto la enzima acetilcolinesterasa (AChE) cerebral como la BChE sanguínea son inhibidas por los $\mathrm{CB}$ y OF. En el presente estudio la inhibición de $\mathrm{BChE}$ fue del $42 \%$ en el grupo intoxicado con respecto al grupo control. Este valor fue semejante al 50\% reportado por Talcott y Peterson (2013) donde los signos clínicos son visibles; sin embargo, en este estudio, todos los animales presentaron signos compatibles con intoxicación, de mayor o menor gravedad. La BChE plasmática es considerada un indicador más sensible que la $\mathrm{AChE}$ en la intoxicación por anticolinesterásicos asociada a los eritrocitos en caninos, al igual que en humanos, ya que suele ser inhibida en mayor grado, incluso antes de que los signos clínicos de la intoxicación aparezcan (Maia et al., 2012).

Una de las intenciones de este trabajo fue de promover en la práctica veterinaria la medición de la colinesterasa en animales intoxicados como herramienta orientativa de la exposición a $\mathrm{OF}$ y $\mathrm{CB}$, insecticidas de mayor frecuencia en este tipo de eventos (Ferré et al., 2015). Asimismo, presentar la posibilidad de aplicar la metodología de cromatografía en capa fina (CCF) (Afful et al., 2008), de baja complejidad y costo que permitió identificar el agente causal. El mayor inconveniente en la aplicación de este método es el uso de solventes y de estándares de plaguicidas peligrosos para la salud de los laboratoristas, por lo que se requiere de una campana de extracción de gases y vapores, así como de personal especializado. Tiene, además, la desventaja de ser un método cualitativo.

Los plaguicidas domésticos, rodenticidas, piretroides y OF son los principales causantes de intoxicación por plaguicidas en humanos en Mendoza (Ferré et al., 2015). Los grupos de plaguicidas agrícolas reportados por el CIAT-Mendoza como principal causa de intoxicación en humanos son los $\mathrm{CB}$ $(24.7 \%)$ y $\operatorname{los}$ OF (24.1\%) (S. Saracco, Mendoza, comunicación personal). Esta información de la población humana respalda el hallazgo de un $\mathrm{CB}$ en el presente evento y otro $\mathrm{CB}$, carbofuran, el año anterior en Mendoza (Ferré et al., 2015). Estos eventos sustentan el hecho de que el uso de determinados plaguicidas en intoxicaciones intencionales en animales es un reflejo del tipo de de plaguicida utilizado en agricultura en la región (Martínez-Haro et al., 2008).

En el Sistema Nacional de Vigilancia Epidemiológica (SINAVE) de Argentina se denuncian las intoxicaciones de personas por plaguicidas, por monóxido de carbono, por medicamentos y por otros tóxicos (Ministerio de Salud, 2011). Por Resolución Nacional N. ${ }^{\circ} 394$ del Ministerio de Salud y Acción Social de 1994, se estableció que la intoxicación por plaguicidas propiamente dicha es un evento de notificación obligatoria en todo el territorio nacional, incorporándose al SINAVE cuando se observara la ocurrencia de brotes. En el caso de la práctica veterinaria, se deberían registrar las denuncias e incluir los datos de los animales afectados, signos clínicos, etc., tal y como se lleva a cabo en otros 
países (Caloni et al., 2016). Esto ayudaría a mejorar el plan de acción en virtud del tratamiento del paciente y efectuar un pronóstico preciso para los propietarios del mismo. El reporte de estos eventos en la casuística veterinaria tiene una implicancia directa en la tarea de control y fiscalización de uso de plaguicidas que pueden tener comercialización prohibida, pero que no han sido aún retirados del mercado.

\section{Agradecimientos}

Al CONICET y a la Universidad Juan Agustín Maza y DICyT (Mendoza) por el financiamiento del trabajo; a la Dra. Raquel Fernández por la supervisión de la pericia toxicológica; al Dr. Sergio Saracco, Director del CIAT- Mendoza; al MV Pablo Vallejos y MV Georgina Hidalgo, propietarios de las clínicas veterinarias de El Bermejo, Guaymallén (Mendoza), que colaboraron activamente en esta investigación; y a los propietarios de los animales por la información proporcionada que permitió estimar la magnitud del evento.

\section{Literatura Citada}

1. Afful S, Dogbe SA, Ahmad K, Ewusie A. T. 2008. Thin layer chromatographic analyses of pesticides in a soil ecosystem. W Africa J Appl Ecol 14: 1-7.

2. Anastasio JD, Sharp CR. 2011. Acute aldicarb toxicity in dogs: 15 cases (20012009). J Vet Emerg Crit Car 21: 253260. doi: 10.1111/j.1476-4431.2011.00613.x

3. Arnot LF, Veale DJH, Steyl JCA, Myburgh JG 2011. Treatment rationale for dogs poisoned with aldicarb (carbamate pesticide). J S Afr Vet Assoc 82:232-238.

4. Berny P, Caloni F, Croubels S, Sachana $M$, Vandenbroucke $V$, Davanzo F, Guitart R. 2010. Animal poisoning in Europe. Part 2: companion animals. Vet J 183: 255-259. doi: 10.1016/ j.tvj1.2009.03.034
5. Cabrera A, Varela W. 2009. Intoxicación por organofosforados (revisión del diagnóstico y manejo). Rev Méd Cos Cen 588: 161-167.

6. Caloni F, Cortinovis C, Rivolta M, Davanzo F. 2016. Suspected poisoning of domestic animals by pesticides. Sci Total Environ 539: 331-336. doi: 10.1016/ j.scitotenv.2015.09.005

7. Campbell A, Chapman M. 2000. Handbook of poisoning in dogs and cats. Oxford: Blackwell Science. 272 p.

8. Cazenave SOS, Itho SF, Lanaro $R$, Chasin AAM. 2005. Aldicarb: uma possibilidade de análise com finalidade forense. Rev Bras Toxicol 18: 105-111.

9. Colovic MB, Krstic DZ, LazarevicPašti TD, Bondzic AM, Vasic VM. 2013. Acetylcholinesterase Inhibitors: pharmacology and toxicology. Curr Neuropharmacol 11: 315-335. doi: 10.2174/1570159X11311030006

10. Ellman GL, Courtney KD, Andres V, Featherstone RM. 1961. A new and rapid colorimetric determination of acetylcholinesterase activity. Biochem Pharmacol 7: 88-91. doi: 10.1016/00062952(61)90145-9

11. Ferré DM, Saldeña EL, Albarracín L, Neuilly V, Gorla NB. 2015. Inhibición de butirilcolinesterasa en dos perros intoxicados y confirmación analítica de carbofuran como agente causal. Rev Vet 26: 43-48.

12. Flanagan RJ, Braithwaite RA, Brown SS, Widdop B, de Wolff FA. 1995. Basic analytical toxicology. Geneva: World Health Organization. $186 \mathrm{p}$.

13. Groner E, Ashani Y, Schorer D, Sterling J, Herzig Y, Weinstock M. 2007. The kinetics of inhibition of human acetylcholinesterase and butyrylcholinesterase by two series of novel carbamates. Mol Pharmacol 71: 1610-1617. doi: 10.1124/mol.107.033928

14. Hynes V. 2014. Protección personal y ambiental en las prácticas de bioseguridad por aplicación de plaguicidas en fincas frutícolas de Mendoza, Argentina, Tesis de Magister. Rosario, Argentina: Universidad Nacional de Rosario. 86 p. 
15. Hopper K, Aldrich J, Haskins SC. 2002. The recognition and treatment of the intermediate syndrome of organophosphate poisoning in a dog. J Vet Emerg Crit Care 12: 99-103. doi: 10.1046/j.1435-6935.2002.00008.x

16. Lima JS, Reis CAG. 1995. Poisoning due to illegal use of carbamates as a rodenticide in Rio de Janeiro. J Toxicol Clin Toxicol 33: 687-690. doi: 10.3109/ 15563659509010629

17. Maia AR, Pérez López M, Soler Rodríguez F. 2012. Comparación de tres métodos de determinación de la actividad colinesterasa plasmática en perro. Rev Toxicol 29: 135-140.

18. Martínez-Haro M, Mateo R, Guitart $R$, Soler-Rodríguez F, Pérez-López M, María-Mojica P, García-Fernández AJ. 2008. Relationship of the toxicity of pesticide formulations and their commercial restrictions with the frequency of animal poisonings. Ecotoxicol Environ Saf 69: 396-402. doi: 10.1016/j.ecoenv.2007.05.006

19. Ministerio de Salud. Presidencia de la Nación. Boletín Integrado de Vigilancia $N .^{\circ} 101 S E$ 50: 76 p. [Internet]: Disponible en: http://www.msal.gob.ar/ images/stories/boletines/BoletinIntegrado-DeVigilanciaVersionVF_SE50.pdf

20. Nelson LS, Perrone J, DeRoos F, Stork C, Hoffman RS .2001. Aldicarb poisoning by an illicit rodenticide imported into the United States: Tres Pacitos. J Toxicol Clin Toxicol 39: 447-452. doi: 10.1081/CLT-100105414

21. Ruiz-Suárez N, Boada LD, Henríquez-Hernández LA, GonzálezMoreo F, Suárez-Pérez A, Camacho M, Zumbado M, et al. 2015. Continued implication of the banned pesticides carbofuran and aldicarb in the poisoning of domestic and wild animals of the Canary Islands (Spain). Sci Total Environ 505: 1093-1099. doi: 10.1016/j.scitotenv. 2014.10.093

22. Sistema Estadístico Municipal. 2001. Censo Nacional de Población, Hogares y Viviendas 2001. [Internet]. Disponible en: http://www.deie.mendoza.gov.ar/publicaciones/publicacionmunicipal/ estadisticasprovinciales/Guaymallen.pdf

23. Soler Rodríguez F, Oropesa AL, Pérez $M .2006$. Análisis de los envenenamientos en fauna silvestre. Situación en Extremadura. Rev Toxicol 23: 35-38.

24. Talcott P, Peterson M. 2013. Small animal toxicology. St. Louis, USA: Elsevier. $930 \mathrm{p}$.

25. Verster RS, Botha CJ, Naidoo V, Van Schalkwyk OL. 2004. Aldicarb poisoning of dogs and cats in Gauteng during 2003. J SAfr Vet Assoc 75: 177 181. doi: $10.4102 /$ jsava.v75i4.479

26. Waseem M, Perry C, Bomann S, Pai M, Gernsheimer J. 2010. Cholinergic crisis after rodenticide poisoning. West J Emerg Med 11: 524-527.

27. [WHO] World Health Organization. 2014. IARC monographs on the evaluation of carcinogenic risks to humans. Report of the Advisory Group to Recommend Priorities for IARC Monographs during 2015-2019. Lyon, France. [Internet]. Disponible en: https:/ /monographs.iarc.fr/ENG/Publications/ internrep/14-002.pdf

28. Xavier FG, Righi DA, Flório JC, Spinosa HS. 2007. Cromatografia em camada delgada para o diagnóstico da intoxicação por aldicarb («chumbinho») em cães e gatos. Arq Bras Med Vet Zootec 59: 1231-1235. doi: 10.1590/ S0102-09352007000500020 